\title{
Study on the Bauschinger effect with increasing of tensile strength in dual phase steel sheets
}

\author{
K. Watanabe ${ }^{1}$, K. Natori ${ }^{1}$, T. Tanaka ${ }^{2}$ \& Y. Imaida ${ }^{2}$ \\ ${ }^{I}$ Graduate School of Engineering, Doshisha University, Japan \\ ${ }^{2}$ Faculty of Science and Engineering, Doshisha University, Japan
}

\begin{abstract}
The purpose of this study is to investigate the deformation property, under a high strain rate, of high strength steel sheets. The influence of strain rate on the Bauschinger effect is clarified, and the relation between the mechanical property and this influence is formulized. In this study, three kinds (590MPa, $780 \mathrm{MPa}$, $980 \mathrm{MPa}$ ) of high strength steel sheets of the Dual Phase type were applied. Both the rolling direction and the transverse direction specimens are created, and the compressive-tensile tests are conducted on three conditions of strain rate $7.0 \times 10^{-3}$, 10 and 100/s. As a result, it is shown clearly in Dual Phase steels that the dependence of strain rate on the Bauschinger effect exists. In addition, with the transformation of material into high strength, it is demonstrated that consideration of the influence of the Bauschinger effect becomes increasingly important; however, the need for consideration of the dependence of the strain rate and the influence of strength anisotropy on the Bauschinger effect decrease for a material model in a numerical simulation.

Keywords: sheet metal forming, mechanical property, compression test, tensile test, high speed deformation, dual phase steel, Bauschinger effect, strain rate dependence, strength anisotropy.
\end{abstract}

\section{Introduction}

In recent years, in connection with the motion in the low carbon society towards earth's environment protection, the improvement in fuel consumption of the automobile has been required strongly. Therefore, adopting high strength steel sheets as the composition material of the automobile body contributed to both the weight reduction of the body and the improvement in safety. It has been 
recognized that Dual Phase steels have more advantages for crash performance than high strength steels, which has same material strength [1].

However, although high strength steel sheets have increasing application in the automobile by the development of material and forming technologies, these steels still present difficulties for processing. The serious problem in press forming was that a spring back occurs. As a solution to this problem, when the forming die was designed, the predicted value of the amount of spring backs by the numerical simulation technique was used. When bending in press forming, sheet metal is influenced by the Bauschinger effect, so, a material model that can consider the Bauschinger effect has been developed in order to improve simulation accuracy [2]. Although consideration of strain rate is important for improvements in productivity, in this model, the influence that it has on the Bauschinger effect is not taken into consideration.

It has been reported in a past study that the dependency of strain rate and strain rate history exist in pure iron and carbon steels [3]. With regard to Dual Phase steels, many data on the Bauschinger effect has been reported [4, 5], while the dependency of strain rate on the effect has been reported in the condition of strain rate from 0.001 to $0.01 / \mathrm{s}$ only [6], which has not been clarified in higher strain rates.

Then, the purpose of this study is to investigate the Bauschinger effect under a high strain rate with regard to high strength steel sheets of the Dual Phase type used due to expectations of weight reduction. To evaluate the Bauschinger effect, re-load tensile tests are conducted after pre compression on conditions of strain rate $7.0 \times 10^{-3}, 10$ and $100 / \mathrm{s}$; the first load process is compression and the second load process is tension. In this paper, the dependence of strain rate on the Bauschinger effect is reported, in addition to the relation between the mechanical property and the scale of the effect.

\section{Bauschinger effect}

When metal materials are applied resulting in stress in a different direction to pre-deformation, yield stress and flow stress decrease significantly compared with the case of stress in the same direction to pre-deformation. This effect has been called the Bauschinger effect. In the case of single phase metal, the back stress arises resulting in long range obstacles, for example piled up dislocation in the cell wall and grain boundary or sessile dislocation; in the reverse stress path, the Bauschinger effect occurs because this back stress helps reversal deformation. In addition, it has been recognized on metal materials containing a hard secondary phase, such as Dual Phase steels, that the Bauschinger effect occurs due to the early yield phenomenon, which is attributed to local strain gradient resulting from the strength differential between the primary and secondary phases $[7,8]$.

Deformation resistance considers two components separately that are independent and dependent on the direction of the stress path. The former is resistance by forest dislocation and yield stress decided by the metal structure, 
and the latter is the back stress. The mean back stress is derived from the following equation [9].

$$
\text { backstress }=\frac{1}{2}\left(\left|\sigma_{\text {reverse }}\right|-\left|\sigma_{\text {reyield } \Delta \varepsilon}\right|\right)
$$

In this study, in order to represent a scale of the Bauschinger effect directly, the ratio of the Bauschinger effect (B.E.R.) is calculated from the following equation [6]. So, the ratio of the Bauschinger effect decreases the rate of the yield point, and is an evaluation indicator in which back stress is made dimensionless by the mechanical property.

$$
\text { B.E.R. }=\frac{\left|\sigma_{\text {reverse }}\right|-\left|\sigma_{\text {reyield } \Delta \varepsilon}\right|}{\left|\sigma_{\text {reverse }}\right|}
$$

Figure 1 depicts the stress values that are used in eqns. (1) and (2). The Bauschinger effect is observed by compressive-tensile tests in this study, and the strain-stress relationship in the tensile process is obtained. $\sigma_{\text {reyield } \Delta \varepsilon}$ is the yield stress in the tensile process, as yield is evaluated at the point at which there is residual plastic strain $\Delta \varepsilon$ is indicated in. $\sigma_{\text {reverse }}$ is the intersection point of the uniaxial tensile history, $\sigma_{\text {tensile }}(\varepsilon)$, with the elastic unloading of compression force history $\sigma_{\text {unload }}(\varepsilon)$, which is calculated as pre-strain $\varepsilon_{\text {pre }}$ and Young's modulus $E$.

B.E.R. as defined eqn. (2) shows the scale of the Bauschinger effect. If $B . E . R$. equals zero, the Bauschinger effect does not occur; the Bauschinger effect occurs more as the value of B.E.R. grows. So, in this study, the evaluation point corresponds to $0.2 \%$ proof stress as yield stress ( $\Delta \varepsilon=0.002)$.

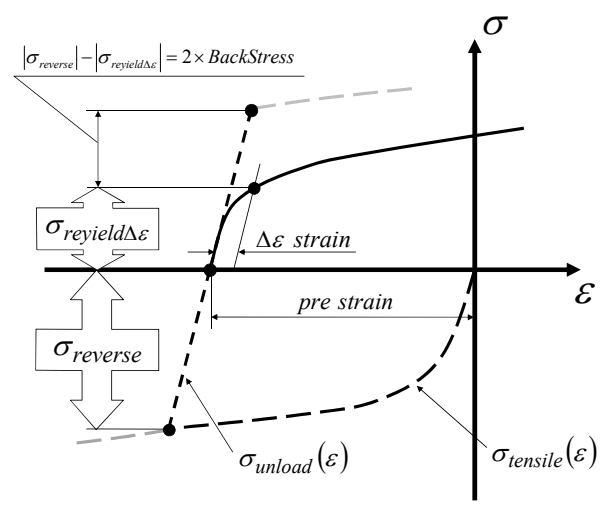

Figure 1: Description of the Bauschinger effect. 
Table 1: The mechanical property of steels. Yield stress is identified as $0.2 \%$ proof stress; $n$ value is calculated as a point of $2 \%$ and $5 \%$ total strain. These data are obtained from the result of a quasi-static tensile test.

\begin{tabular}{|c|c|c|c|c|}
\hline \multicolumn{2}{|c|}{ Rolling Direction } & \multirow[b]{2}{*}{$\begin{array}{l}\text { Yield stress } \\
{[\mathrm{MPa}]}\end{array}$} & \multirow[b]{2}{*}{$\begin{array}{c}\text { Tensile strength } \\
{[\mathrm{MPa}]}\end{array}$} & \multirow[b]{2}{*}{$\begin{array}{c}n \text { value } \\
(2,5 \% \text { total strain })\end{array}$} \\
\hline $\begin{array}{c}\text { RD } \\
\text { samples } \\
\sqrt{n} \\
\end{array}$ & $\begin{array}{c}\text { TD } \\
\text { samples }\end{array}$ & & & \\
\hline \multirow{2}{*}{ DP590 } & $\mathrm{RD}$ & 360 & 609 & 0.193 \\
\hline & TD & 291 & 585 & 0.222 \\
\hline \multirow{2}{*}{ DP780 } & RD & 518 & 853 & 0.153 \\
\hline & TD & 446 & 806 & 0.168 \\
\hline \multirow{2}{*}{ DP980 } & $\mathrm{RD}$ & 716 & 1064 & 0.146 \\
\hline & TD & 553 & 1032 & 0.176 \\
\hline
\end{tabular}

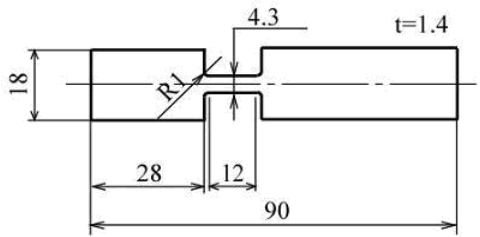

Figure 2: Configuration of specimen.

\section{Experimental}

\subsection{Material and specimens}

The material is cold rolling high strength steel sheets of the Dual Phase type, which contain ferrite and martensite. Dual Phase steels of 590, 780 and $980 \mathrm{MPa}$ classified for strength (DP590, DP780 and DP980) are used. The mechanical property of the steels is listed in table 1 . The configuration of the specimen is shown in fig. 2 [10]. The specimen axis is cut along the rolling direction (RD samples) and along the transverse direction (TD samples) by a wire electrical discharge machine. The constricted part of specimens is called the parallel part.

\subsection{Compression test}

Quasi-static (0.007/s) and dynamic (10, 100/s) compression tests were conducted. A universal testing machine was used for the quasi-static test, and an electro hydraulic high speed compression testing machine was used for the dynamic test. To avoid buckling of the specimen at compression, a compression test jig, which has been developed in this study, was used for the specimen shown in fig.3. The compression test jig is made up of a main part (fig.3(a)) and a support part (fig.3(b)). A specimen is fixed by bolting the support part on to the 
main part, while the specimen fits in the channel of the support part. Then, the specimen is compressed from above with a T-pusher. The amount of pre-strain is $1 \sim 9 \%$, which is adjusted by pusher dimension $L$. So, for the dynamic compression test, the impact power is cushioned by setting the compression test jig in the base with a damper.

We measured the size of the specimen parallel part (width $w_{0}, w$ and thickness $\left.t_{0}, t\right)$ by micrometer before and after compression, respectively. One of the measurement results after compression is shown in fig.4. Specimens are nearly constant in cross sectional dimensions in the axial direction as shown in fig.4, therefore, it is confirmed that compression strain can be applied equally using the developed test jig. The compression pre-strain is calculated from the following equation.

$$
\varepsilon_{\text {pre }}=\ln \frac{w t}{w_{0} t_{0}}
$$

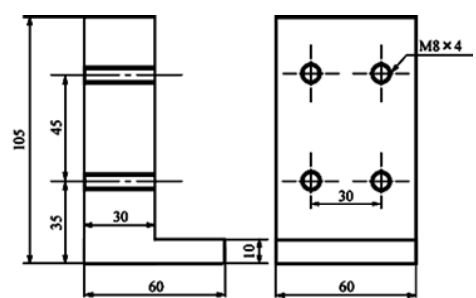

(a)

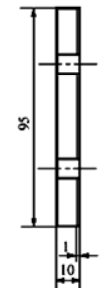

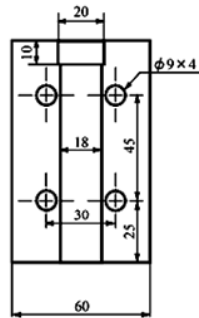

(b)

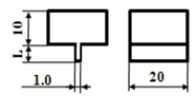

(c)

Figure 3: Configuration of the compression jig, which is separated three parts: (a) main part; (b) support part; (c) pusher.

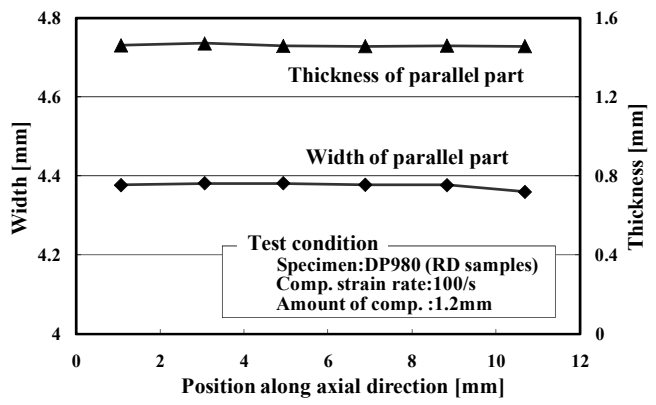

Figure 4: The changes of width and thickness after compression. Notice that compression strain is applied equally. 

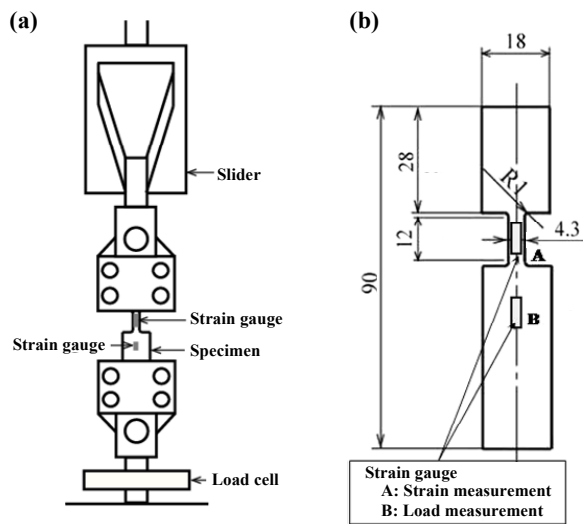

Figure 5: The method for the dynamic tensile test: (a) dynamic tensile test machine; (b) the position of the strain gauge on the specimen.

\subsection{High speed tensile test}

Quasi-static (0.007/s) and dynamic (10, 100/s) tensile tests were conducted after compression. The universal testing machine was used for the quasi-static test, and the electro hydraulic high speed tensile testing machine was used for the dynamic test. A schematic diagram of the dynamic tensile test machine is shown in fig.5(a). This machine's actuator was controlled by an electro hydraulic high speed servo valve as an upper chuck run up at the slider part, therefore, specimens have caused strain at the expectant speed [11].

The most important issue revealed in the high speed tensile test is that wave shapes by the load-cell, including vibration noise, were detected. Vibrations occur from the inertial force of system, including load-cell, chuck and a specimen. So, to reduce the noise effect by the reflected wave, it is necessary that loads at high speed deformation are measured by a strain gauge adapting grip part of a specimen in both sides as shown in fig.5(b) [10]. In addition, to measure the conversion factor from strain to load, we compared data from loads cell to the output of the strain gauge in the quasi-static tensile test.

Strain can be measured by the strain gauge, adapting the parallel part up to about 5\%. Strain exceeding this measurement range was calculated from the mean strain rate, measured by analyzing a motion picture recorded with a high speed camera.

\section{Results and discussion}

\subsection{The dependence of strain rate history on the Bauschinger effect}

Strain rate history dependence on the Bauschinger effect is considered from the result of compressive-tensile tests under the condition that strain rate differs in compressive and tensile processes. The true stress-true strain diagram for RD samples in the tensile process is shown in fig. 6 . 
There are differences in flow stress from differing tensile strain rate; it is considered that these differences occur from the strain rate dependence of stress. On the other hand, it is demonstrated that there are no differences in flow stress if the tensile strain rate is the same, allowing that the pre-compression strain rate is different. In addition, the relation between the compression pre-strain and stress when producing $0.2 \%$ plastic strain is shown in fig.7. Considering $0.2 \%$ proof stress, differences in differing compression pre-strain rate are very small, as is flow stress. Therefore, it is demonstrated that the dependence of strain rate in pre-deformation, or strain rate history dependence, on the Bauschinger effect in Dual Phase steels is negligibly small.

Assuming that the Bauschinger effect occurs by evolution of dislocation substructures, such as cell structure, it is expected that back stress is different, because the configuration of these formed structures differs depending on the strain rate at pre-strain [12]. However, it is considered in this discussion that the influence of cell structure as a factor that causes the Bauschinger effect is small, and cells do not evolve in the pre-compression condition of this study.

\subsection{The dependence of strain rate on the Bauschinger effect}

Strain rate dependence on the Bauschinger effect is considered from the result of compressive-tensile tests under the condition that strain rate is equal in both
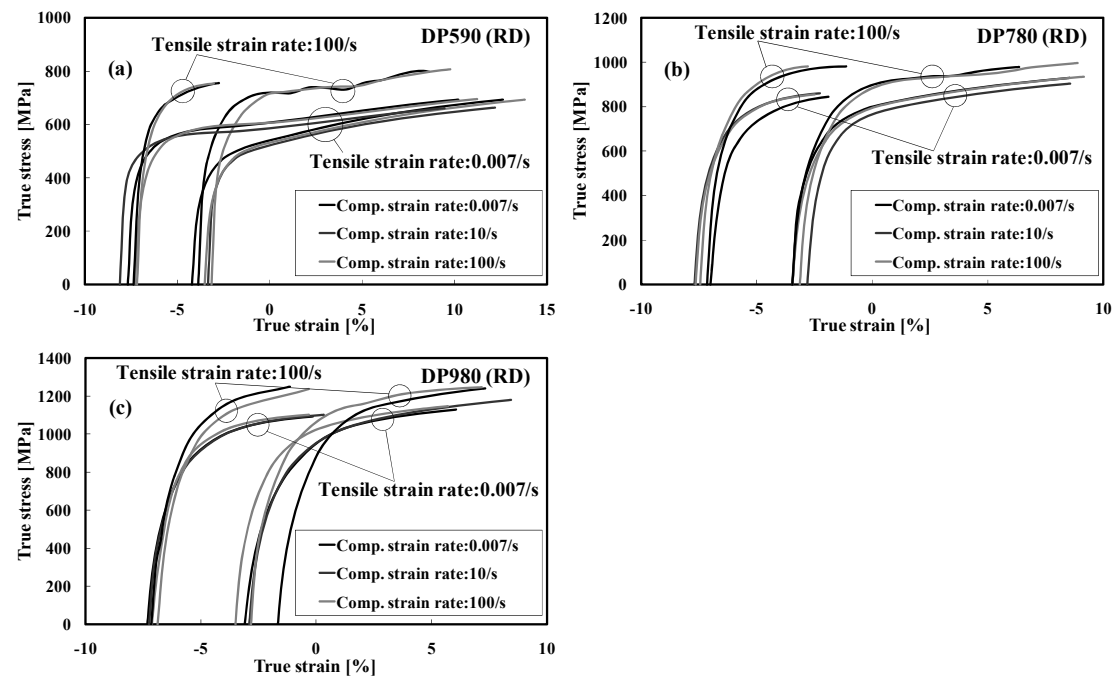

Figure 6: Comparison of the difference in compression strain rate and tensile strain rate: (a) DP590, (b) DP780, (c) DP980 for RD samples. Notice that there are no differences in flow stress by differing compression strain rate, while there are differences by differing tensile strain rate. 
126 High Performance Structures and Materials V
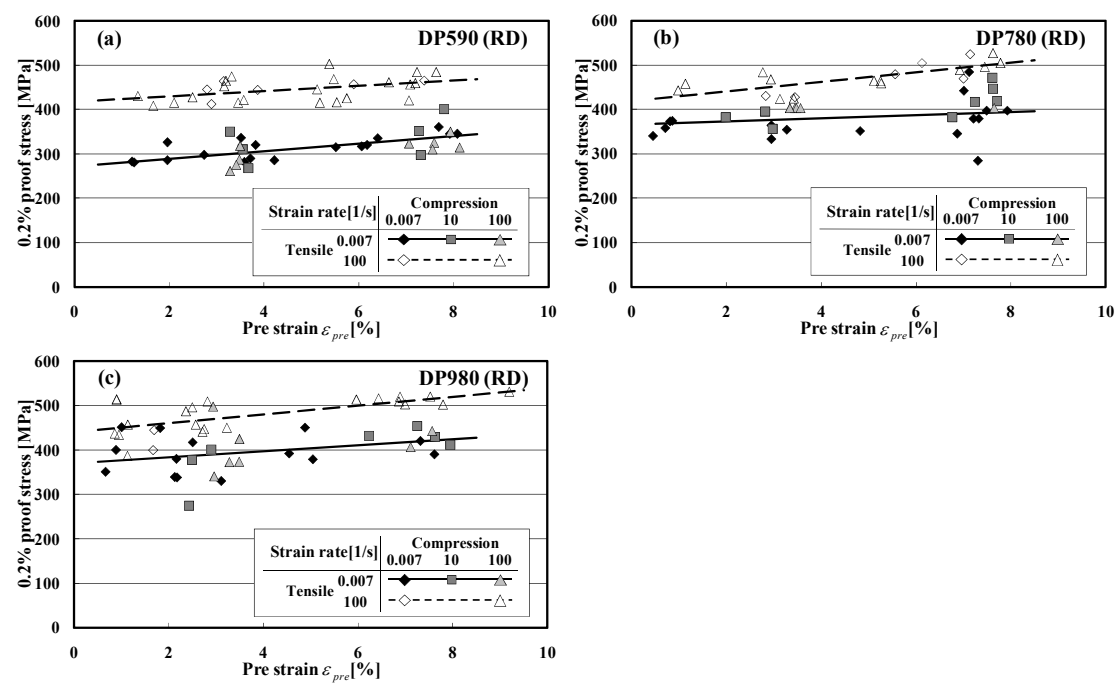

Figure 7: Re-yield stress at the various test condition: (a) DP590, (b) DP780, (c) DP980 for RD samples. The results indicate a tendency similar to that shown in fig.6.
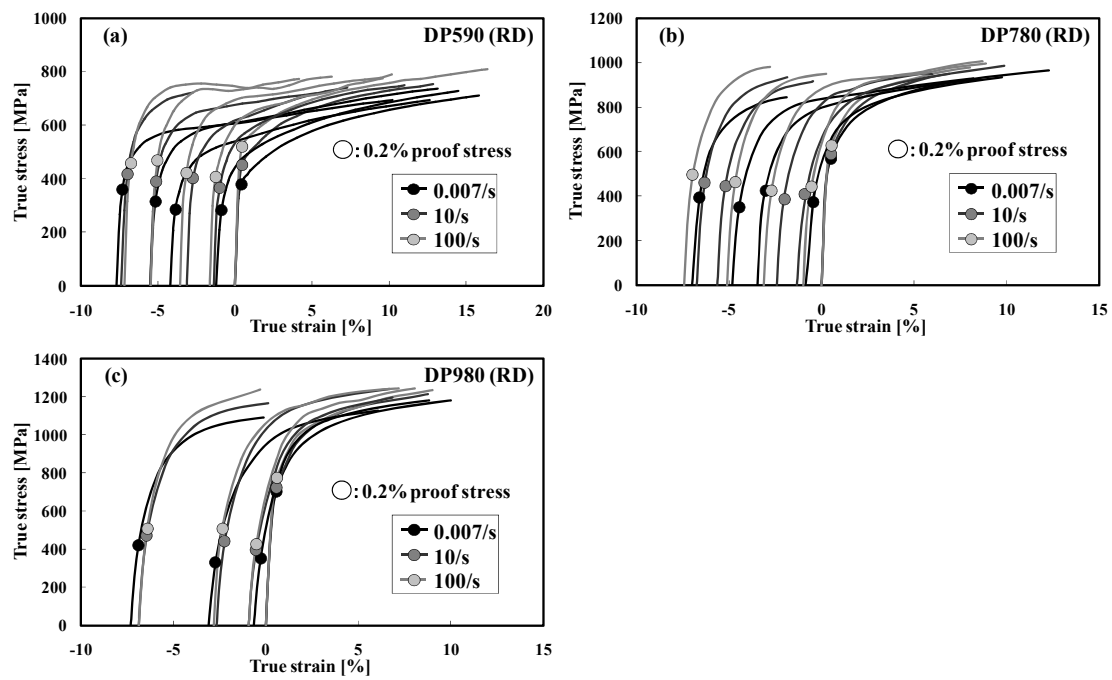

Figure 8: Tensile test result at the same strain rate of compression and tension: (a) DP590, (b) DP780, (c) DP980 for RD samples. 
compressive and tensile process. The true stress-true strain diagram and the added $0.2 \%$ proof stress point for RD samples in the tensile process is shown in fig.8.

Flow stress and $0.2 \%$ proof stress resulting from re-load tensile test decrease significantly compared with the result of the uniaxial tensile test, and as the strain rate increases, these stresses rise at the same compression pre-strain. These suggest occurrence of the Bauschinger effect and the strain rate dependence of stress.

To evaluate the Bauschinger effect quantitatively, the ratio of the Bauschinger effect is calculated from the result of the re-load tensile test and the uniaxial tensile test by eqn. (2). In each material and strain rate, the relation between compression pre-strain and the ratio of the Bauschinger effect is shown in fig.9; approximate curves fitted to this relation by the following equation is added in fig.9.

$$
\text { B.E.R. }=k_{\text {B.E.R. }} \cdot \varepsilon_{\text {pre }}{ }^{n_{\text {B.E.R. }}}, \varepsilon_{\text {pre }} \geq \Delta \varepsilon=0.002
$$

Figure 9 indicates that the ratio of the Bauschinger effect decreases with the increase in strain rate for all materials. Therefore, it is demonstrated that the dependence of strain rate on the Bauschinger effect exists in Dual Phase steel and is correlated negatively with strain rate.

So, it is considered that the ratio of the Bauschinger effect is expressed as a function of compression pre-strain and strain rate. As coefficients $k_{\text {B.E.R. }}$ and $n_{B . E . R}$. of eqn. (4) are considered as a function of strain rate $\dot{\varepsilon}$, the relation
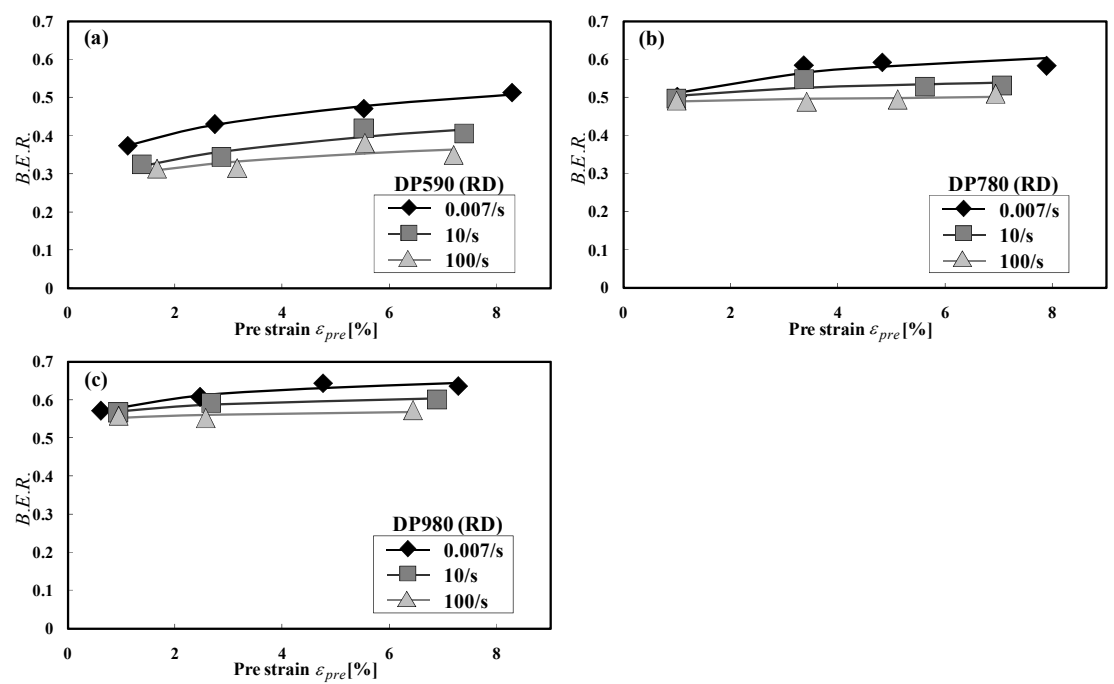

Figure 9: The ratio of the Bauschinger effect: (a) DP590, (b) DP780, (c) DP980 for RD samples. Notice that the Bauschinger effect has the dependence of strain rate. 

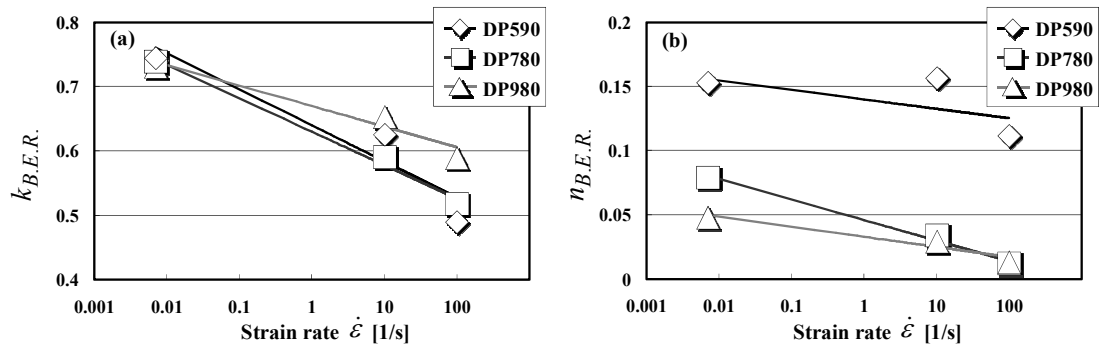

Figure 10: Correlation between strain rate $\dot{\varepsilon}$ and (a) $k_{\text {B.E.R. }}$ or (b) $n_{B . E . R \text {. for }}$ RD samples.

Table 2: $\quad$ Coefficients of eqns. (4) and (5).

\begin{tabular}{|c|c|c|c|c|}
\hline RD samples & $a_{k}$ & $a_{n}$ & $b_{k}$ & $b_{n}$ \\
\hline DP590 & $-5.6 \times 10^{-2}$ & $-7.3 \times 10^{-3}$ & 0.64 & 0.14 \\
\hline DP780 & $-5.2 \times 10^{-2}$ & $-1.6 \times 10^{-2}$ & 0.63 & 0.046 \\
\hline DP980 & $-3.2 \times 10^{-2}$ & $-7.9 \times 10^{-3}$ & 0.67 & 0.033 \\
\hline
\end{tabular}

between strain rate and these coefficients is shown in fig.10(a), (b), respectively. $k_{\text {B.E.R. }}, n_{\text {B.E.R. }}$ is found to correlate with strain rate $\dot{\varepsilon}$ logarithmically as shown in fig.10. Therefore, the dependence of strain rate on the Bauschinger effect is expressed in the following equation by fitting coefficients of eqn. (4).

$$
\begin{aligned}
& k_{\text {B.E.R. }}=a_{k} \log _{10} \dot{\varepsilon}+b_{k} \\
& n_{\text {B.E.R. }}=a_{n} \log _{10} \dot{\varepsilon}+b_{n}
\end{aligned}
$$

New coefficients using eqns. (5) and (6) are constants specific to the material. The constants of each material are listed in table 2. So, according to eqns. (4), (5) and (6), yield stress is expressed in the following equation when materials are applied to re-load after deforming total pre-strain $\varepsilon$ on the stress path of the first and second load process to become in the opposite direction.

$$
\left|\sigma_{\text {reyield } \Delta \varepsilon}\right|=\left\{1-\left(a_{k} \log _{10}+b_{k}\right)\left(|\varepsilon|-\frac{\left|\sigma_{\text {reverse }}\right|}{E}\right)^{\left(a_{n} \log _{10}+b_{n}\right)}\right\}\left|\sigma_{\text {reverse }}\right|
$$

\subsection{The influence of strength anisotropy on the Bauschinger effect}

Specimen materials are as received in this study; strength anisotropy exists as shown in table 1. So, the influence of strength anisotropy on the mechanism of the Bauschinger effect is considered. The quasi-static tensile test is conducted after pre-deforming in the compression strain rate of three conditions for RD and TD samples; the ratio of the Bauschinger effect is calculated from the result by eqn. (1) and the relation between that and pre-strain is shown in fig. 11. 

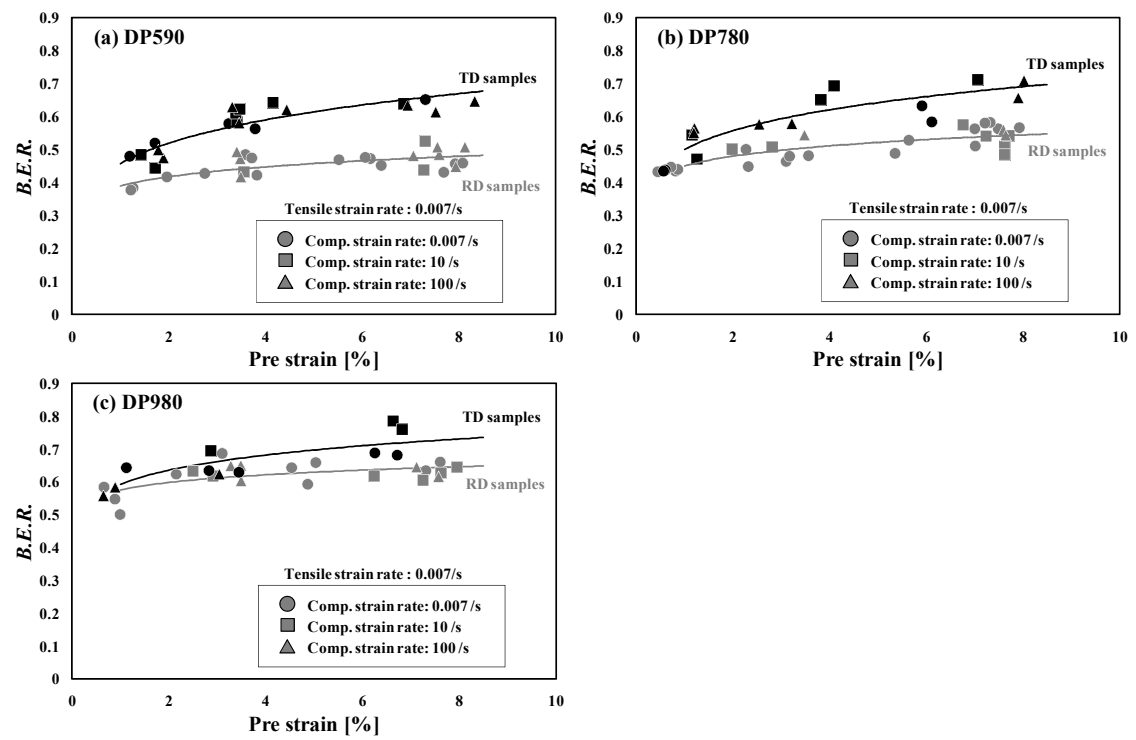

Figure 11: The ratio of the Bauschinger effect in the case of $0.007 / \mathrm{s}$ tensile strain rate: (a) DP590, (b) DP780, (c) DP980. Notice that the Bauschinger effect is influenced by material anisotropy.

Distinctions of the ratio of the Bauschinger effect stress occur by the difference in loading axial direction as shown in fig.11. It has been recognized that strength anisotropy is induced by a mixture of elongated metal structures along the rolling direction, formed crystal texture by controlling crystal lattice, and so on. It is demonstrated in this experimental result that the mechanism of the Bauschinger effect is influenced by these geometric deviations of material texture, which cause strength anisotropy.

\subsection{The relation between material strength and the Bauschinger effect}

The influence of material strength on the Bauschinger effect is considered. In Dual Phase steels, transforming materials into high strength has been controlled by an increase in martensite volume fraction, grain refinement, and so on. In addition, RD samples are stronger than TD samples for strength anisotropy as shown in table 1 , it is suggested that the strength increase due to strength anisotropy is apparent in a reduction in strength differential between the soft ferrite phase and the hard martensite phase resulting from the strength increase of each phase and non-uniformity of the grain size. Coefficients of eqn. (4) are calculated from the results shown in fig.11 toward compression pre-strain; the relation between these and material strength is shown in fig. 12 .

It is indicated that $k_{\text {B.E.R. }}$ is the scale of the Bauschinger effect, and $n_{\text {B.E.R. }}$ is the dependence of the pre-strain amount on the Bauschinger effect. With increasing material strength by transforming materials into high strength or 


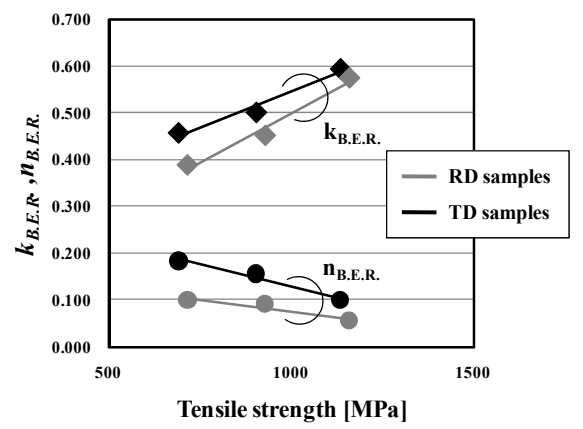

Figure 12: Correlation between $\mathrm{k}$ value or $\mathrm{n}$ value and the mechanical property of steel in the case of $0.007 / \mathrm{s}$ tensile strain rate.

strength anisotropy, it is demonstrated that the dependence of the pre-strain amount decreases, as shown in fig.12. In addition, it is demonstrated that the scale of the Bauschinger effect increases with transforming materials into high strength and on the other hand decreases with increasing material strength by anisotropy. In addition, with transforming materials into high strength, it is demonstrated that the dependence of strain rate and the influence of strength anisotropy on the Bauschinger effect decrease.

The Bauschinger effect occurs because back stress arising as a result of piled up dislocation in the grain boundary helped reversal deformation in the reverse stress path. Furthermore, the Bauschinger effect in Dual Phase steels occurs due to the early yield phenomenon attributed to the local strain gradient, resulting from the strength differential between the primary and secondary phases. The trend of experimental results as referred to above can be explained with these mechanisms of the Bauschinger effect.

\section{Conclusion}

In this study, to clarify the influence of strain rate on the Bauschinger effect on high strength steel sheets of the Dual Phase type, the method of compressivetensile testing for sheet metal under high speeds has been developed. To evaluate the Bauschinger effect, a compressive-tensile test has been conducted in a wide range of strain rates from quasi-static to dynamic in the case of experimental conditions in which compressive and tensile axial directions were the same and along the RD or the TD in Dual Phase steels. The results indicate that the dependence of strain rate is correlated negatively with strain rate. In addition, the reduction of strength resulting from the Bauschinger effect is formulized to prestrain and strain rate.

In the future, it has been expected that further application into high strength steels will be promoted. In order to apply high strength steels, it is demonstrated that consideration of the influence of the Bauschinger effect becomes increasingly important, but the need for consideration of the dependence of strain 
rate and the influence of strength anisotropy on the Bauschinger effect decrease for the material model in a numerical simulation. In addition, even for the same strength of material, it is estimated that the mechanism of the Bauschinger effect is different due to the difference in microstructure of the materials.

\section{References}

[1] Urabe, T. \& Hosoya, Y., J. Soc. Tech. Plasticity, 46(534), pp. 16-20, 2005.

[2] Yoshida, F. \& Uemori, T., Int. J. Mech. Sci., 45(10), pp.1687-1702, 2003.

[3] Hasebe, T., Proc. of the 16th Computational Mechanics Conf. on Japan Soc. Mech. Eng., pp.415-416, 2003.

[4] Uko, D., Sowerby, R. \& Embury, J. D., Metals. Tech., 7, pp.359-367, 1980.

[5] Goel, A., Ray, R. K.\& Murty, G. S., Scr. Metall., 17(3), pp.375-380, 1983.

[6] Han, K., Vantyne, C. J. \& Levy, B. S., Metal. Mater. Trans. A, 36A(5), pp.2379-2384, 2005.

[7] Yakou, T. \& Hasegawa, T., Tetsu-to-Hagane, 70(11), pp.51-58, 1984.

[8] Matsui, K., Terada, K., Akiyama, M., Kuboki, T. \& Oikawa, K., Trans. Japan Soc. Mach. Eng. A, 68(675), pp.71-78, 2002.

[9] Mori, Y., Ashida, U., Kishi, T. \& Horiuchi, R., Tetsu-to-Hagane, 64(9), pp.1414-1423, 1978.

[10] Mukai, Y. \& Watanabe, K., Kobe Steel Eng. Report, 52(3), pp.32-34, 2002.

[11] Takii, T., Inspection Eng., 18(11), pp.51-56, 2003.

[12] Hasebe, T., Proc. of the 15th Computational Mechanics Conf. on Japan Soc. Mech. Eng., pp.137-138, 2002. 